\title{
Association between Parathyroid Hormone, 25 (OH) Vitamin D, and Chronic Kidney Disease: A Population-Based Study
}

\author{
Wei-Hao Wang, ${ }^{1}$ Li-Wei Chen, ${ }^{2,3,4}$ Chin-Chan Lee, ${ }^{1,3,4}$ Chiao-Yin Sun, ${ }^{1,3,4}$ \\ Yu-Chiau Shyu, ${ }^{4}$ Heng-Rong Hsu, ${ }^{1,3,4}$ Rong-Nang Chien, ${ }^{2,3,4}$ and I-Wen Wu ${ }^{1,3,4}$ \\ ${ }^{1}$ Department of Nephrology, Keelung Chang Gung Memorial Hospital, Keelung, Taiwan \\ ${ }^{2}$ Department of Gastroenterology and Hepatology, Keelung Chang Gung Memorial Hospital, Keelung, Taiwan \\ ${ }^{3}$ College of Medicine, Chang Gung University, Taoyuan, Taiwan \\ ${ }^{4}$ Community Medicine Research Center, Keelung Chang Gung Memorial Hospital, Keelung, Taiwan
}

Correspondence should be addressed to I-Wen Wu; fliawu@yahoo.com

Received 4 November 2016; Revised 25 January 2017; Accepted 14 February 2017; Published 7 March 2017

Academic Editor: You-Ying Chau

Copyright (c) 2017 Wei-Hao Wang et al. This is an open access article distributed under the Creative Commons Attribution License, which permits unrestricted use, distribution, and reproduction in any medium, provided the original work is properly cited.

\begin{abstract}
Identification of the accurate risk factor for CKD remains mandatory to combat the high prevalence of diseases. Growing evidence suggests the association of serum vitamin $\mathrm{D}$ with diverse health conditions. However, the relationship between vitamin $\mathrm{D}$, intact parathyroid hormone $(\mathrm{PTH})$, and calcium-phosphate metabolism and development of CKD remains controversial. We conduct this cross-sectional observational study to investigate the association between serum $25(\mathrm{OH})$ vitamin $\mathrm{D}$, intact PTH, and calcium and phosphate levels with eGFR and albuminuria, as a surrogate marker of CKD, in a community population. A total of 4080 participants were recruited. The mean age was $58.4 \pm 13.3$ years and $1480(36.3 \%)$ were men. The mean eGFR was $94.1 \pm 26.3 \mathrm{~mL} / \mathrm{min} / 1.73 \mathrm{~m}{ }^{2}$. The prevalence of CKD was $19.8 \%$. Serum $25(\mathrm{OH})$ vitamin D and log intact PTH levels were inversely correlated with eGFR but positively correlated with log albuminuria. Logistic regression analysis identified the log intact PTH as an independent factor associated with eGFR $\leq 60 \mathrm{~mL} / \mathrm{min} / 1.73 \mathrm{~m}^{2}$ and proteinuria. This association was consistent when serum intact PTH was analyzed as continuous as well as categorical variables (as hyperparathyroidism). The relationship remains significant using resampling subset analysis with comparable baseline characteristics and adjustment for $25(\mathrm{OH})$ vitamin $\mathrm{D}$, calcium, and phosphate levels. This finding warranted further research to clarify the causal relationship of PTH/25 (OH) vitamin D with the risk of CKD in the general population.
\end{abstract}

\section{Introduction}

Chronic kidney disease (CKD) is a worldwide public health issue because of its wide association with multiple comorbidities, demanding high cardiovascular events, mortality, and expensive medical cost. The worldwide prevalence of CKD ranged from 8 to $16 \%$, dependent on the disease definition, study design, and racial groups [1]. The estimated national prevalence of CKD is $11.9 \%$ [2] in Taiwan, which has the highest incidence and prevalence of end-stage renal disease (ESRD) worldwide [3]. Ascertainment of accurate risk factors associated with CKD is mandatory to allow timely diagnosis and intervention to decrease the burden of the disease. The associations of CKD with several traditional risk factors (such as hypertension, diabetes, obesity, smoking, dyslipidemia, and metabolic syndrome) were well established in the literature [4]. Epidemiological literatures emphasized the association between serum vitamin $\mathrm{D}$ level and nonskeletal disease as cardiovascular events [5-7], metabolic syndrome [8], cancer $[9,10]$, autoimmune disease [11], and critical illness [12]. The decrease of glomerular filtration rate restricts delivery of substrate to the 1-alpha-hydroxylase and decreases the production of $1,25(\mathrm{OH})$ vitamin $\mathrm{D}$ by the kidney in CKD patients. The interplay between vitamin D, fibroblast growth factor-23 (FGF-23), intact parathyroid hormone (PTH), and calcium-phosphorus-bone metabolism is disrupted with the progression of renal function [1315]. However, the relationship between vitamin $D$, intact PTH, and calcium-phosphate metabolism and development of CKD remains controversial. 
A Korean population-based study found a biphasic change of serum vitamin D levels, according to CKD severity. The estimated glomerular filtration rate (eGFR) was negatively associated with serum vitamin D levels [16] in the entire population; however, the mean vitamin $\mathrm{D}$ values were decreased with the lowering of eGFR levels in moderate and severe CKD stages [16, 17]. A Swiss population study did not find any association between serum vitamin D levels and CKD or albuminuria [18]. In addition, the association between vitamin $\mathrm{D}$ levels and renal function decline was lost after adjustment for baseline eGFR [19]. Accumulating evidence indicated the association of abnormal calcium-phosphate metabolism with cardiovascular disease in the general population. Intact PTH levels were associated with hypertension [20] and abnormal calciumphosphate metabolism was associated with coronary artery calcification [21]. The prevalence ratio of intact PTH among participants of the US National Health and Nutrition Examination Survey showed a stepwise increment of 2.30 and 4.69 for individuals with an eGFR of 45 to 59 and 30 to $44 \mathrm{~mL} / \mathrm{min} / 1.73 \mathrm{~m}^{2}$ compared with those people having eGFR $>$ or $=60 \mathrm{~mL} / \mathrm{min} / 1.73 \mathrm{~m}^{2}$ [22]. While the serum value variation and the relationship between vitamin $\mathrm{D} /$ intact $\mathrm{PTH}$ and hard outcomes were evident in moderate to late stage CKD and ESRD patients, little is known about the exact role of vitamin $\mathrm{D}$, intact $\mathrm{PTH}$, and calcium and phosphate levels all together in the risk of CKD in healthy subjects.

We conduct this cross-sectional observational study to investigate the association between serum $25(\mathrm{OH})$ vitamin $\mathrm{D}$, intact PTH, and calcium and phosphate levels with eGFR and albuminuria and the risk of CKD in a community population.

\section{Method}

2.1. Patient Setting and Data Description. Participants of community health activity from August 2013 to May 2016 in the northeastern region of Taiwan were enrolled in the study. Participants aged greater than 30 years and who were not pregnant were included after obtaining informed consent $(n=4916)$. Individuals who had received vitamin D supplementation or any over-the-counter vitamin supplements $(n=830)$ or incomplete baseline biochemistry data $(n=6)$ were excluded from the analysis. Demographic data were assessed from questionnaires. Anthropometric and biochemistry measurements were performed at the entry of the study. Blood samples were obtained after an overnight fast, and the following parameters were determined: complete blood cell count, liver and renal biochemistry parameters, lipid profiles, fasting sugar, and intact PTH and total 25 $(\mathrm{OH})$ vitamin D levels. This study was approved by the ethics committee of the institutional review board of the Keelung Chang Gung Memorial Hospital.

2.2. Definitions. CKD were defined by the National Kidney Foundation: K/DOQI classification for CKD and were determined as having persistent proteinuria or a decreased eGFR of less than $60 \mathrm{~mL} / \mathrm{min} / 1.73 \mathrm{~m} 2$, determined by the abbreviated Modification of Diet in Renal Disease equation [23]. Serum Cr was assessed by spectrophotometric analysis using a modified kinetic Jaffe reaction with standardization of the creatinine calibration to an isotope dilution mass spectrometry reference measurement procedure. Proteinuria was determined if urine albumin-to-creatinine ratio $>30 \mathrm{~g} / \mathrm{g}$ or urine protein-to-creatinine ratio $>150 \mathrm{~g} / \mathrm{g}$. Diabetes mellitus was defined as a fasting glucose level $\geq 126 \mathrm{mg} / \mathrm{dL}$ or use of any hypoglycemic medication. Hypertension was considered present if the patient received medical therapy for such a condition or if blood pressure was $>140 / 90 \mathrm{mmHg}$. Hypercholesterolemia was defined as a total cholesterol level $\geq 200 \mathrm{mg} / \mathrm{dL}$. Smoking and alcohol drinking indicated any sustained past or current behaviors. Metabolic syndrome was defined according to the Adult Treatment Panel III criteria as the presence of at least three of the following five traits: visceral (abdominal) obesity, determined on the basis of the Asian waist circumference cut-offs (men: $>90 \mathrm{~cm}$, women: $>80 \mathrm{~cm}$ ); blood pressure $>130 / 85 \mathrm{mmHg}$ or drug treatment for essential hypertension; serum high-density lipoprotein cholesterol (HDL-C) level $<40 \mathrm{mg} / \mathrm{dL}(1 \mathrm{mmol} / \mathrm{L})$ in men and $<50 \mathrm{mg} / \mathrm{dL}(1.3 \mathrm{mmol} / \mathrm{L})$ in women or drug treatment for low HDL-C; serum triglycerides (TG) level $>150 \mathrm{mg} / \mathrm{dL}$ $(1.7 \mathrm{mmol} / \mathrm{L})$ or drug treatment for elevated TG; and fasting plasma glucose level $>100 \mathrm{mg} / \mathrm{dL}(5.6 \mathrm{mmol} / \mathrm{L})$ or drug treatment for DM. Obesity was evaluated according to the WHO classification as having a body mass index of $30 \mathrm{~kg} / \mathrm{m} 2$ or more [24]. Serum concentrations of $25(\mathrm{OH})$ vitamin D were measured using a radioimmunoassay (Vitamin D Total, Roche Diagnostics, Mannheim, Germany) according to the manufacturer's instructions. Vitamin D status was defined as "deficient" (<20 ng/mL), "insufficient" (20-30 ng/mL), and "sufficient" (>30 ng/mL) [25]. Hypocalcemia was defined as corrected Ca less than $8.5 \mathrm{mg} / \mathrm{dL}$; hyperphosphatemia, $p$ greater than $4.5 \mathrm{mg} / \mathrm{dL}$, or phosphate binder use and hyperparathyroidism, intact parathyroid hormone more than twice the upper limit of normal, corresponding to $70 \mathrm{pg} / \mathrm{mL}$ [26]. Because of the cross-sectional nature of the study and to avoid misdiagnosis of $\mathrm{CKD}$, we used two surrogate indices of CKD (eGFR $<60 \mathrm{~mL} / \mathrm{min}$ and proteinuria) to establish the association between the biomarker of interest and the outcome.

2.3. Statistical Methods. Descriptive statistics were expressed as mean \pm standard deviation, median, range, or percentage frequency, as appropriate. All variables were tested for normal distribution by Kolmogorov-Smirnov test. Data were logtransformed to approximate normal distribution. Student's $t$-test or Mann-Whitney $U$ test was applied to compare the mean of continuous variables. Categorical data were tested using the Chi-square test. Pearson or Spearman correlation coefficients were appropriately used to test the correlation between serum $25(\mathrm{OH})$ vitamin $\mathrm{D}$, intact $\mathrm{PTH}$, and calcium and phosphate levels with eGFR or proteinuria. Logistic regression analysis was applied to identify the association between these variables with the outcome of interest, after adjusting for potential confounders, such as age or gender. Conditional logistic regression analysis was performed to 


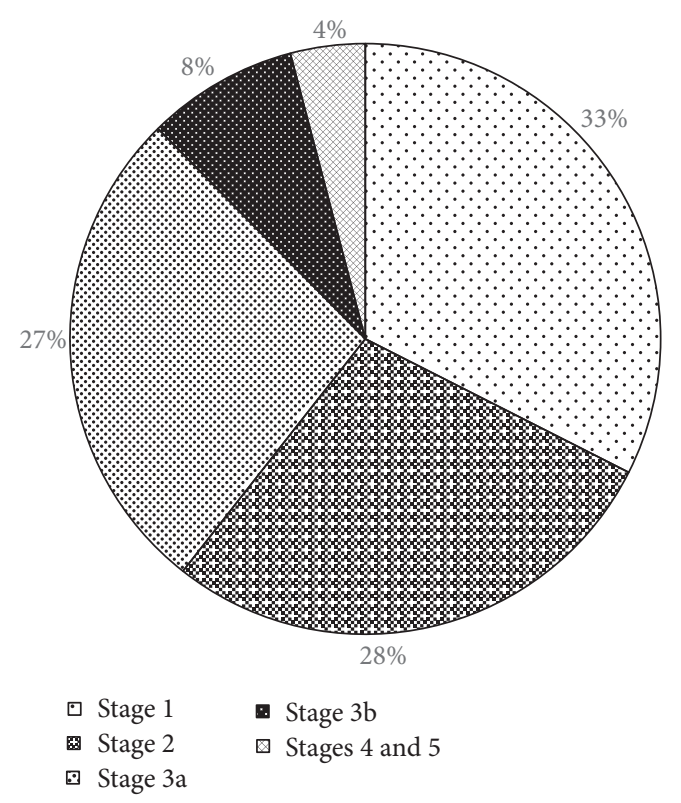

FIGURE 1: Distribution of CKD stage of the entire population.

evaluate the odd ratio of factors associated with outcome in the resampling subset, matched by age ( \pm 1 year old), gender, CKD stage, presence of hypertension, and metabolic syndrome, at 1:2 ratio. All statistical tests were two-tailed, and a $p<0.05$ was considered statistically significant. Data were analyzed using SPSS 17.0 for Windows (SPSS Inc., Chicago, IL).

\section{Results}

A total of 4080 participants were included in the analysis. The mean age was $58.4 \pm 13.3$ years and 1480 (36.3\%) were men. The mean eGFR of the study population was $94.1 \pm$ $26.3 \mathrm{~mL} / \mathrm{min} / 1.73 \mathrm{~m}^{2}$. Table 1 lists the baseline characteristics of all participants and between two groups. The overall prevalence of CKD was $19.8 \%$. Figure 1 illustrates the distribution of CKD stage of the entire population. CKD patients were more likely to be older, to be male, and to be obese. They were more likely to have diabetes, hypertension, metabolic syndrome, and smoking/drinking habits. CKD patients had higher serum $25(\mathrm{OH})$ vitamin $\mathrm{D}[31.1 \pm 10.5 \mathrm{ug} / \mathrm{mL}$ versus $29.0 \pm 9.0 \mathrm{ug} / \mathrm{mL}, p<0.001]$ and higher intact PTH $[46.3$ $(8.3,898.9) \mathrm{pmol} / \mathrm{L}$ versus $42.2(3.0,311.2) \mathrm{pmol} / \mathrm{L}, p<$ 0.001] levels than normals (Table 1). Table 2 summarizes serum $25(\mathrm{OH})$ vitamin D status stratified by eGFR levels and albuminuria-to-creatinine ratio. Serum $25(\mathrm{OH})$ vitamin $\mathrm{D}(r=-0.212, p<0.001), \log$ intact PTH $(r=-0.121$, $p<0.001)$, and calcium $(r=-0.079, p<0.001)$ were inversely correlated with eGFR, while phosphate $(r=0.116$, $p<0.001)$ was positively correlated with eGFR. However, serum $25(\mathrm{OH})$ vitamin $\mathrm{D}(r=0.016, p=0.259), \log$ intact PTH $(r=0.142, p<0.001)$, calcium $(r=0.038$, $p=0.030)$, and phosphate $(r=0.005, p=0.665)$ were positively correlated with log albuminuria-to-creatinine ratio (Figure 2). Figure 3 depicts the trajectory of these biomarkers with eGFR. Logistic regression analysis identified the log intact PTH as an independent factor associated with CKD [crude: odd ratios (OR), 1.985; 95\% confidential interval (CI), 1.663-2.371, $p<0.001$; model 1 (adjusted for age and gender): OR, 1.618; 95\% CI, 1.345-1.948, $p<0.001$; model 2 (adjusted for age, gender, $25(\mathrm{OH})$ vitamin $\mathrm{D}$, calcium, and phosphate): OR, 1.796, 95\% CI, 1.479-2.181, $p<0.001]$. The association of intact PTH with CKD was consistent when assessed in a categorical fashion, as hyperphosphatemia [crude: OR, 2.530; 95\% CI, 2.057-3.112, $p<0.001$; model 1 (adjusted for age and gender): OR, 2.074; 95\% CI, 1.662-2.589, $p<0.001$; model 2 (adjusted for age, gender, $25(\mathrm{OH})$ vitamin D status, hypocalcemia, and hyperphosphatemia): OR, 2.128, 95\% CI, 1.699-2.667, $p<0.001$ (Table 3)]. Again, log intact PTH was an independent factor associated with proteinuria [crude: OR, 1.744, 95\% CI, 1.442-2.109, $p<0.001$; model 1 (adjusted for age and gender): OR, 1.477, 95\% CI, 1.217-1.793, $p<0.001$; model 2 (adjusted for age, gender, $25(\mathrm{OH})$ vitamin D status, hypocalcemia, and hyperphosphatemia): OR, 1.579, 95\% CI, 1.291-1.932, $p<0.001]$. The association of intact PTH with proteinuria was consistent when assessed in a categorical fashion, as hyperphosphatemia [crude: OR, 2.212, 95\% CI, $1.770-2.766, p<0.001$; model 1 (adjusted for age and gender): OR, 1.865, 95\% CI, 1.480-2.351, $p<0.001$; model 2 (adjusted for age, gender, $25(\mathrm{OH})$ vitamin $\mathrm{D}$ status, hypocalcemia, and hyperphosphatemia): OR, 1.895, 95\% CI, 1.497-2.398, $p<$ 0.001 (Table 4)]. The associations of serum $25(\mathrm{OH})$ vitamin $\mathrm{D}$, calcium, and phosphate levels with $\mathrm{CKD}$ or proteinuria were not significant after adjusting for confounder or analysis in a categorical manner (Tables 3 and 4 ).

To control as much as possible the confounding effect of baseline characteristics of patients on the outcome of study, we have resampled a subset of patients from stratified sampling by CKD stage with individualized match to age, gender, presence of hypertension, or metabolic syndrome. Stratified sampling by CKD stage identified 464 CKD patients with their matched counterparts ( $n=928$ normals). Only 1392 patients were included in the subset analysis after individualized matched pair of $\pm 1 \mathrm{y} / \mathrm{o}$ of age. The mean age of resampling subset was $61.0 \pm 10.9$ years and $39 \%$ of them were men. Hypertension was present in $73 \%$ and metabolic syndrome was present in $43 \%$ of the resampling population. Both the log intact PTH and hyperparathyroidism remained a significant risk factor for eGFR $\leq 60 \mathrm{~mL} / \mathrm{min}$ and albuminuria, after adjustment for age, gender, serum $25(\mathrm{OH})$ vitamin $\mathrm{D}$, and calcium or phosphate levels (Table 5).

\section{Discussion}

This community-based study found that the serum PTH levels were inversely correlated with eGFR but positively correlated with albuminuria. Either the increase of serum PTH levels or the presence of hyperparathyroidism was independently associated with the risk of CKD, after adjustments for confounders or by using the resampling subset of comparable baseline characteristics. The serum PTH levels increased in the early stage of CKD, before the change of 25 $(\mathrm{OH})$ vitamin $\mathrm{D}$-calcium-phosphate axis, and may serve as a potential risk factor for CKD. 

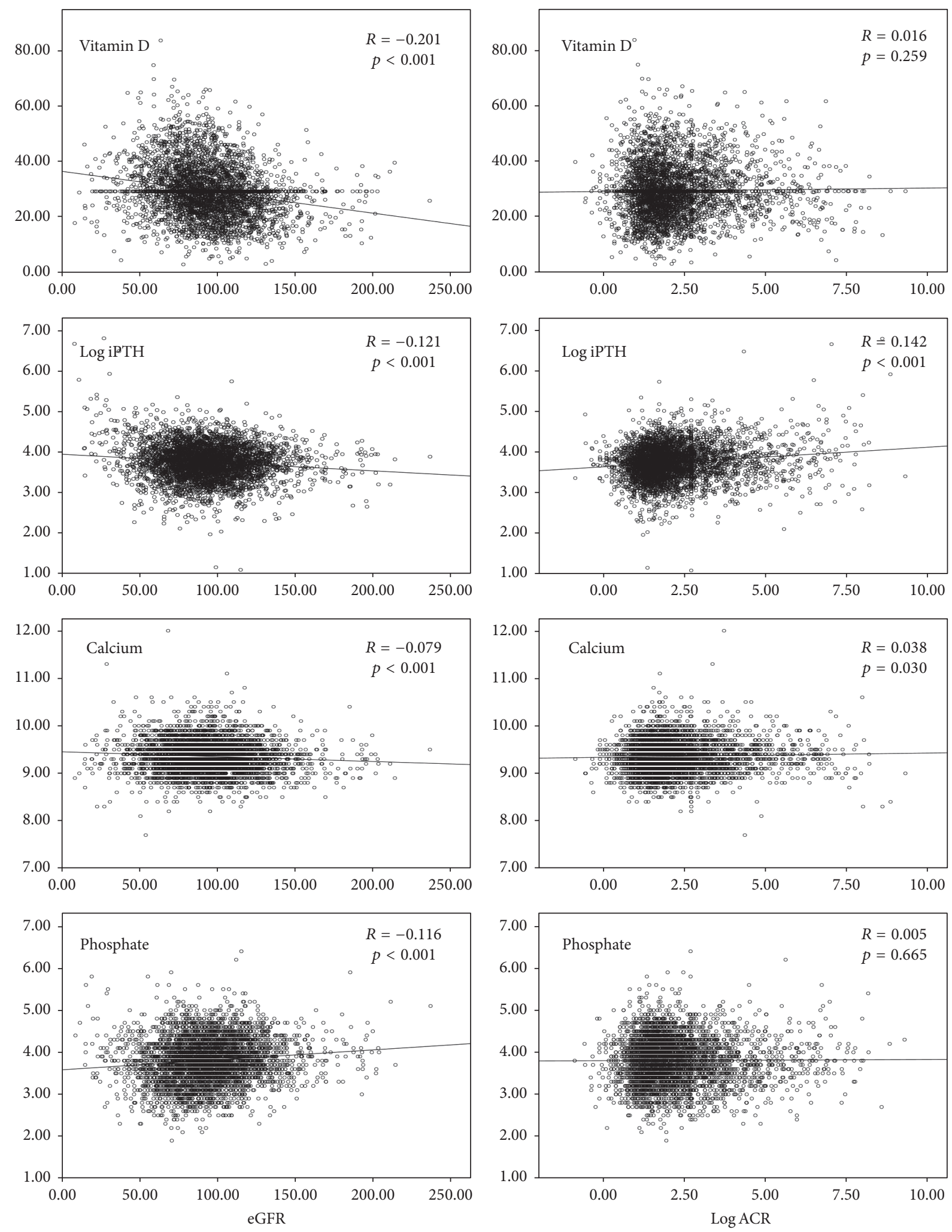

FIGURE 2: Correlation of serum $25(\mathrm{OH})$ vitamin D, log intact PTH, and calcium and phosphate with eGFR and log albuminuria-to-creatinine ratio. 
TABLE 1: Demographic characteristics of all patients and stratified by CKD $(n=4080)$.

\begin{tabular}{|c|c|c|c|c|}
\hline & $\begin{array}{c}\text { All } \\
(n=4080)\end{array}$ & $\begin{array}{l}\text { Non-CKD } \\
(n=3273)\end{array}$ & $\begin{array}{c}\text { CKD } \\
(n=807)\end{array}$ & $p$ \\
\hline Age, years & $58.4 \pm 13.3$ & $56.5 \pm 12.6$ & $66.1 \pm 12.9$ & $<0.001$ \\
\hline Male, number (\%) & $1480(36.3 \%)$ & $1134(34.6 \%)$ & $346(42.9 \%)$ & $<0.001$ \\
\hline Diabetes, number (\%) & $722(17.7 \%)$ & $410(12.5 \%)$ & $312(38.7 \%)$ & $<0.001$ \\
\hline Hypertension, number (\%) & $2197(53.8 \%)$ & $1607(49.3 \%)$ & $590(73.2 \%)$ & $<0.001$ \\
\hline Metabolic syndrome, number (\%) & $1277(31.3 \%)$ & $863(26.4 \%)$ & $414(51.3 \%)$ & $<0.001$ \\
\hline Obesity, number (\%) & $383(9.4 \%)$ & $264(8.1 \%)$ & $119(14.7 \%)$ & $<0.001$ \\
\hline Physical activity, min/day & $12.9(0,660)$ & $12.9(0,660)$ & $17.1(0,600)$ & 0.137 \\
\hline Smoking, number (\%) & $1032(25.3 \%)$ & $806(24.6 \%)$ & $226(28.0 \%)$ & 0.048 \\
\hline Alcohol drinking, number (\%) & $642(15.7 \%)$ & $534(16.3 \%)$ & $108(13.4 \%)$ & 0.040 \\
\hline Body mass index, $\mathrm{kg} / \mathrm{m}^{2}$ & $24.9 \pm 3.8$ & $24.6 \pm 3.7$ & $26.0 \pm 3.8$ & $<0.001$ \\
\hline Systolic BP, mmHg & $131.9 \pm 19.4$ & $129.6 \pm 18.5$ & $141.3 \pm 20.1$ & $<0.001$ \\
\hline Diastolic BP, mmHg & $78.5 \pm 11.9$ & $77.7 \pm 11.5$ & $81.9 \pm 12.8$ & $<0.001$ \\
\hline \multicolumn{5}{|l|}{ Laboratory } \\
\hline eGFR, $\mathrm{mL} / \mathrm{min}$ per $1.73 \mathrm{~m}^{2}$ (MDRD) & $94.1 \pm 26.3$ & $98.3 \pm 22.6$ & $77.1 \pm 32.6$ & $<0.001$ \\
\hline BUN, mg/dL & $13.7 \pm 5.5$ & $12.7 \pm 3.7$ & $17.5 \pm 8.9$ & $<0.001$ \\
\hline Serum creatinine, $\mathrm{mg} / \mathrm{dL}$ & $0.8 \pm 0.3$ & $0.7 \pm 0.2$ & $1.0 \pm 0.5$ & $<0.001$ \\
\hline Serum albumin, g/dL & $4.7 \pm 0.3$ & $4.7 \pm 0.3$ & $4.6 \pm 0.3$ & $<0.001$ \\
\hline Cholesterol, mg/dL & $209.7 \pm 39.2$ & $210.1 \pm 37.4$ & $208.2 \pm 45.8$ & 0.209 \\
\hline Triglycerides, mg/dL & $128.4 \pm 114.4$ & $121.4 \pm 103.3$ & $156.5 \pm 148.1$ & $<0.001$ \\
\hline hs-CRP, mg/L & $1.0(0.2,211.4)$ & $0.9(0.2,104.1)$ & $1.6(0.2,211.4)$ & $<0.001$ \\
\hline Urine albumin-to-creatinine ratio, $\mathrm{g} / \mathrm{g}$ & $6.5(0.4,11067.4)$ & $5.4(0.6,29.8)$ & $49.7(0.4,11067.4)$ & $<0.001$ \\
\hline $25(\mathrm{OH})$ vitamin $\mathrm{D}, \mathrm{ug} / \mathrm{mL}$ & $29.4 \pm 9.4$ & $29.0 \pm 9.0$ & $31.1 \pm 10.5$ & $<0.001$ \\
\hline iPTH, pmol/L & $43.0(3.0,898.9)$ & $42.2(3.0,311.2)$ & $46.3(8.3,898.9)$ & $<0.001$ \\
\hline Serum calcium, mg/dL & $9.4 \pm 0.3$ & $9.4 \pm 0.3$ & $9.4 \pm 0.4$ & 0.055 \\
\hline Corrected calcium, mg/dL & $8.8 \pm 0.3$ & $8.8 \pm 0.3$ & $8.9 \pm 0.4$ & $<0.001$ \\
\hline Serum phosphate, mg/dL & $3.8 \pm 0.5$ & $3.8 \pm 0.5$ & $3.8 \pm 0.6$ & 0.003 \\
\hline $25(\mathrm{OH})$ vitamin $\mathrm{D}$ status & & & & $<0.001$ \\
\hline Vitamin D sufficient, number (\%) & $1383(33.9 \%)$ & $1042(31.8 \%)$ & $341(42.3 \%)$ & \\
\hline Vitamin D deficient, number (\%) & $2124(52.1 \%)$ & $1755(53.6 \%)$ & $369(45.7 \%)$ & \\
\hline Vitamin D insufficient, number (\%) & $573(14.0 \%)$ & $476(14.5 \%)$ & $97(12.0 \%)$ & \\
\hline
\end{tabular}

The values are expressed as means (SD) or median (min, max).

Corrected calcium $=$ serum calcium $+0.8 *(4-$ serum albumin $)$.

TABLE 2: Serum $25(\mathrm{OH})$ vitamin D status stratified by eGFR and albuminuria-to-creatinine ratio.

\begin{tabular}{lccc}
\hline Variable & $\begin{array}{c}\text { Vitamin D sufficient, } \\
\text { number (\%) }\end{array}$ & $\begin{array}{c}\text { Vitamin D deficient, } \\
\text { number (\%) }\end{array}$ & $\begin{array}{c}\text { Vitamin D insufficient, } \\
\text { number (\%) }\end{array}$ \\
\hline Mean eGFR, mL/min & $607(27.2 \%)$ & $1227(55.0 \%)$ & $398(17.8 \%)$ \\
eGFR $>$ 90 mL/min & $619(40.5 \%)$ & $772(50.5 \%)$ & $139(9.1 \%)$ \\
eGFR 90-60 mL/min & $118(54.1 \%)$ & $79(36.2 \%)$ & $21(9.6 \%)$ \\
eGFR 60-45 mL/min & $39(39.0 \%)$ & $46(46 \%)$ & $15(15 \%)$ \\
eGFR $<45 \mathrm{~mL} / \mathrm{min}$ & & & $497(14.2 \%)$ \\
Mean ACR ratio, g/g & $1147(32.8 \%)$ & $1851(53.0 \%)$ & $44(9.4 \%)$ \\
ACR $<30$ & $203(43.5 \%)$ & $220(47.1 \%)$ & $32(27.1 \%)$ \\
ACR 30-300 & $33(28.0 \%)$ & $53(44.9 \%)$ & 0.2586 \\
ACR $>300$ & & & \\
\hline
\end{tabular}

eGFR: estimated glomerular filtration rate; ACR: urine albumin-to-creatinine ratio. 
TABLE 3: OR of variables associated with eGFR less than $60 \mathrm{~mL} / \mathrm{min}$.

\begin{tabular}{|c|c|c|c|c|c|c|}
\hline \multirow{2}{*}{ Variable } & \multicolumn{2}{|l|}{ Crude } & \multicolumn{2}{|l|}{ Model 1} & \multicolumn{2}{|l|}{ Model 2} \\
\hline & OR $(95 \% \mathrm{CI})$ & $p$ & OR $(95 \% \mathrm{CI})$ & $p$ & OR $(95 \% \mathrm{CI})$ & $p$ \\
\hline \multicolumn{7}{|l|}{ Continuous variables } \\
\hline $25(\mathrm{OH})$ vitamin $\mathrm{D}, \mathrm{ug} / \mathrm{mL}$ & $1.023(1.015-1.031)$ & $<0.001$ & $1.001(0.992-1.010)$ & 0.795 & $1.005(0.996-1.015)^{*}$ & 0.276 \\
\hline Log intact PTH, pmol/L & $1.985(1.663-2.371)$ & $<0.001$ & $1.618(1.345-1.948)$ & $<0.001$ & $1.796(1.479-2.181)^{*}$ & $<0.001$ \\
\hline Calcium, mg/dL & $1.276(1.020-1.597)$ & 0.033 & $1.475(1.168-1.862)$ & 0.001 & $1.617(1.270-2.060)^{*}$ & $<0.001$ \\
\hline Phosphate, mg/dL & $0.802(0.694-0.926)$ & 0.003 & $1.086(0.919-1.283)$ & 0.332 & $1.138(0.959-1.350)^{*}$ & 0.137 \\
\hline \multicolumn{7}{|l|}{ Categorical variables } \\
\hline Vitamin D sufficient (yes versus no) & 1 & & 1 & & 1 & \\
\hline Vitamin D deficient (yes versus no) & $0.642(0.544-0.758)$ & $<0.001$ & $0.872(0.662-1.149)$ & 0.330 & $0.959(0.725-1.269)^{\#}$ & 0.771 \\
\hline Vitamin D insufficient (yes versus no) & $0.623(0.485-0.800)$ & $<0.001$ & $0.726(0.558-0.944)$ & 0.017 & $0.777(0.595-1.014)^{\#}$ & 0.063 \\
\hline Hyperparathyroidism (yes versus no) & $2.530(2.057-3.112)$ & $<0.001$ & $2.074(1.662-2.589)$ & $<0.001$ & $2.128(1.699-2.667)^{\#}$ & $<0.001$ \\
\hline Hypocalcemia (yes versus no) & $1.236(0.529-2.892)$ & 0.624 & $0.940(0.374-2.360)$ & 0.896 & $0.659(0.261-1.665)^{\#}$ & 0.378 \\
\hline Hyperphosphatemia (yes versus no) & $0.935(0.728-1.200)$ & 0.598 & $1.237(0.948-1.612)$ & 0.117 & $1.298(0.994-1.696)^{\#}$ & 0.560 \\
\hline
\end{tabular}

OR: odd ratios; CI: confidence interval.

Model 1: adjusted for age and gender.

Model 2: * adjusted for age, gender, and all other continuous variables; ${ }^{*}$ adjusted for age, gender, and all other categorical variables.

TABLE 4: OR of variables associated with proteinuria.

\begin{tabular}{|c|c|c|c|c|c|c|}
\hline \multirow{2}{*}{ Variable } & \multicolumn{2}{|l|}{ Crude } & \multicolumn{2}{|l|}{ Model 1} & \multicolumn{2}{|l|}{ Model 2} \\
\hline & OR $(95 \% \mathrm{CI})$ & $p$ & OR $(95 \% \mathrm{CI})$ & $p$ & OR $(95 \% \mathrm{CI})$ & $p$ \\
\hline \multicolumn{7}{|l|}{ Continuous variables } \\
\hline $25(\mathrm{OH})$ vitamin $\mathrm{D}, \mathrm{ug} / \mathrm{mL}$ & $1.016(1.008-1.025)$ & $<0.001$ & $1.000(0.991-1.010)$ & 0.941 & $1.004(0.994-1.014)^{*}$ & 0.457 \\
\hline Log intact PTH, pmol/L & $1.744(1.442-2.109)$ & $<0.001$ & $1.477(1.217-1.793)$ & $<0.001$ & $1.579(1.291-1.932)^{*}$ & $<0.001$ \\
\hline Calcium, mg/dL & $1.159(0.907-1.481)$ & 0.237 & $1.252(0.980-1.599)$ & 0.072 & $1.330(1.037-1.705)^{*}$ & 0.025 \\
\hline Phosphate, mg/dL & $0.865(0.740-1.012)$ & 0.069 & $1.070(0.897-1.276)$ & 0.452 & $1.118(0.935-1.338)^{*}$ & 0.222 \\
\hline \multicolumn{7}{|l|}{ Categorical variables } \\
\hline Vitamin D sufficient (yes versus no) & 1 & & 1 & & 1 & \\
\hline Vitamin D deficient (yes versus no) & $1.386(1.060-1.813)$ & 0.017 & $0.887(0.666-1.183)$ & 0.416 & $0.963(0.720-1.289)^{\#}$ & 0.801 \\
\hline Vitamin D insufficient (yes versus no) & $0.971(0.747-1.263)$ & 0.826 & $0.756(0.576-0.992)$ & 0.044 & $0.802(0.609-1.056)^{\#}$ & 0.115 \\
\hline Hyperparathyroidism (yes versus no) & $2.212(1.770-2.766)$ & $<0.001$ & $1.865(1.480-2.351)$ & $<0.001$ & $1.895(1.497-2.398)^{\#}$ & $<0.001$ \\
\hline Hypocalcemia (yes versus no) & $1.332(0.542-3.272)$ & 0.532 & $1.109(0.436-2.821)$ & 0.827 & $0.821(0.320-2.107)^{\#}$ & 0.682 \\
\hline Hyperphosphatemia (yes versus no) & $0.992(0.759-1.298)$ & 0.955 & $1.201(0.909-1.587)$ & 0.198 & $1.249(0.943-1.653)^{\#}$ & 0.120 \\
\hline
\end{tabular}

OR: odd ratios; CI: confidence interval.

Model 1: adjusted for age and gender.

Model 2: * adjusted for age, gender, and all other continuous variables; ${ }^{*}$ adjusted for age, gender, and all other categorical variables.

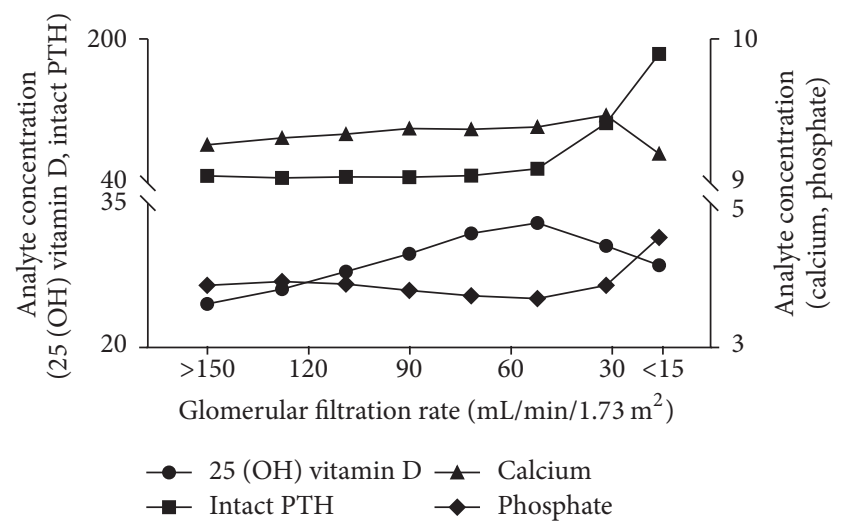

FIGURE 3: Trajectory of serum $25(\mathrm{OH})$ vitamin $\mathrm{D}$, intact PTH, and calcium and phosphate with eGFR.
Optimal serum levels of vitamin D in healthy subjects and CKD patients are not well understood. Growing evidence indicated the relationship between abnormal vitamin $\mathrm{D}$ levels and metabolic syndrome, diabetes, cancer, and chronic diseases; however, the association of vitamin $\mathrm{D}$ and the risk of CKD remains controversial [27]. Our findings that serum $25(\mathrm{OH})$ vitamin D levels increased with the decline of eGFR and the insignificant association with CKD are consistent with some studies $[16,18,19]$, but not with others $[28,29]$. Guessous et al. found that the prevalence of serum vitamin $\mathrm{D}$ concentration and deficiency status were similar in CKD and non-CKD subjects in a cross-sectional Swiss population-based study [18]. They did not find any significant association of serum vitamin $\mathrm{D}$ levels with incident CKD, incident albuminuria, or rapid renal function decline, after a mean follow-up of 5.5 years [19]. On the other hand, 
TABLE 5: Conditional logistic regression analysis in resampling subset of patients.

\begin{tabular}{|c|c|c|c|c|}
\hline \multirow{2}{*}{ Variable } & \multicolumn{2}{|c|}{$\mathrm{eGFR} \leq 60 \mathrm{~mL} / \mathrm{min} / 1.73 \mathrm{~m}^{2}$} & \multicolumn{2}{|c|}{ Proteinuria } \\
\hline & Adjusted OR (95\% CI) & $p$ & Adjusted OR (95\% CI) & $p$ \\
\hline \multicolumn{5}{|l|}{ Continuous variables } \\
\hline $25(\mathrm{OH})$ vitamin $\mathrm{D}, \mathrm{ug} / \mathrm{mL}$ & $1.012(0.999-1.025)^{*}$ & 0.079 & $1.006(0.993-1.020)^{*}$ & 0.380 \\
\hline Log intact PTH, pmol/L & $1.613(1.240-2.099)^{*}$ & $<0.001$ & $1.471(1.117-1.938)^{*}$ & 0.006 \\
\hline Calcium, mg/dL & $1.421(1.003-2.014)^{*}$ & 0.048 & $1.044(0.722-1.510)^{*}$ & 0.818 \\
\hline Phosphate, mg/dL & $1.155(0.913-1.463)^{*}$ & 0.23 & $1.231(0.962-1.576)^{*}$ & 0.098 \\
\hline \multicolumn{5}{|l|}{ Categorical variables } \\
\hline Vitamin D sufficient (yes versus no) & 1 & & 1 & \\
\hline Vitamin D deficient (yes versus no) & $1.323(0.896-1.953)^{\#}$ & 0.16 & $1.096(0.735-1.634)^{\#}$ & 0.653 \\
\hline Vitamin D insufficient (yes versus no) & $0.989(0.685-1.427)^{\#}$ & 0.951 & $0.887(0.611-1.290)^{\#}$ & 0.531 \\
\hline Hyperparathyroidism (yes versus no) & $1.994(1.442-2.758)^{\#}$ & $<0.001$ & $1.836(1.313-2.567)^{\#}$ & $<0.001$ \\
\hline Hypocalcemia (yes versus no) & $0.167(0.020-1.373)^{\#}$ & 0.096 & $0.232(0.028-1.920)^{\#}$ & 0.176 \\
\hline Hyperphosphatemia (yes versus no) & $1.677(1.162-2.421)^{\#}$ & 0.006 & $1.611(1.105-2.347)^{\#}$ & 0.013 \\
\hline
\end{tabular}

OR: odd ratios; CI: confidence interval; eGFR: estimated glomerular filtration rate.

*Adjusted for age, gender, and all other continuous variables. ${ }^{*}$ Adjusted for age, gender, and all other categorical variables.

findings from the 10-year Prevention of Renal and Vascular End-Stage Disease prospective cohort demonstrated that low plasma vitamin D was associated with the risk of developing albuminuria rather than reduced eGFR in high sodium intake individuals [28]. However, investigation on normal renal function subjects found that high serum vitamin D levels were independently associated with high serum creatinine and recalled the potential role of muscle size as a contributing factor to this elevation [29]. Several factors can influence serum levels of vitamin $\mathrm{D}$, including analytic method, seasonal variation, latitude, air pollution, sun exposure, and physical activity [30]. Furthermore, meta-analyses to evaluate the concentration of vitamin $\mathrm{D}$ and health outcome did not show convincing data for the association of this biomarker with renal outcome in healthy or CKD patients [27].

The finding that serum PTH levels and hyperparathyroidism were independent risk factors for CKD and its surrogates (eGFR and proteinuria) was novel. Molecularly, parathyroid hormone is considered as procalcific and profibrotic through promoting mRNA and protein expression of the receptor of advanced glycation end products (RAGE) and interleukin 6, enhancing abnormal calcium-phosphate homeostasis and renin-angiotensinogen-aldosterone hyperactivity; even some have advocated its possible roles as cardiovascular or uremic toxins [31-33]. On the other hand, treatment of teriparatide (human PTH) in lowdensity lipoprotein receptor $-/-$ mice exhibited induction of osseous osteopontin expression and serum osteopontin levels, indicating inhibition of vascular calcification and aortic osteogenic differentiation. The study suggested possible beneficial actions of PTH at early stages of macrovascular disease in responses to diabetes and dyslipidemia [34]. Clinically, a Swedish population-based study showed that normocalcemic, vitamin D sufficient hyperparathyroidism was common and indolent in a long-term follow-up of 17 years. This condition triggered low morbidities and had no association with creatinine at follow-up [35]. However, data of the Germany Calcific Uraemic Arteriolopathy Registry indicated development of calciphylaxis in dialysis patients with mean PTH levels of 147 (IQR: 72-276) pg/mL [36]. In spite of the promising results of our study, caution in the interpretation of PTH as a powerful biomarker should be taken into account, including intermethod and interpersonal variability, target metabolite to measure, vitamin D status, baseline renal function, age, gender, menopausal status, body mass index, race, and end-organ hyporesponsiveness [3740]. Further studies are needed to demonstrate the exact relationship between high intact PTH and development of $\mathrm{CKD}$ in the general population.

A peculiar finding of the present study was the elevated odd ratio of having eGFR $<60 \mathrm{~mL} / \mathrm{min}$ associated with serum calcium levels, even after considering confounders. However, this association was no longer existent when the serum calcium level was assessed in a categorical manner. Physiologically, serum calcium levels decrease with the decline of eGFR. This phenomenon appears in the late stage of CKD and contributes in part to the hyperparathyroidism cascade leading to the development of CKD-bone mineral disease and adverse patient outcomes. Conversely, high serum calcium levels promote neointimal calcification and increase cardiovascular events and mortality. The paradoxical role of serum calcium levels was rarely explored in healthy or early stage CKD patients and is unclear. Association of high corrected serum calcium with both the number of the metabolic syndrome components and the number of nonconventional cardiometabolic risk factors (uric acid, homocystein, and gamma-glutamyltransferase) was observed in Caucasian people, independently of the metabolic syndrome and body mass index [41]. A French multicentric study found that increased serum calcium concentration was independently and positively associated with high pulse pressure and hypertension [42], an important risk factor for CKD. Putting all these lines of evidence together, the exact impact of serum calcium levels on the renal functions of healthy people deserves further study. 
The strength of our study was in the use of a large number of participants with high range for renal function, simultaneous measurement of biochemistry parameters that minimize bias in the variability of analytic testing, and exclusion of exogenous vitamin D use. The use of subset sampling analysis of comparable characteristics has avoided confounding effects from baseline data on the study outcome. However, some limitations of study should be addressed. First, findings from the cross-sectional design study should not be interpreted in casual terms. Second, all participants of the study came from the northeastern region of Taiwan (Keelung and its neighboring areas) and had unique geographic (latitude, $25^{\circ} \mathrm{N}$ ), cultural, and dietary characteristics that limited generalizability of the present findings to other populations. Finally, important drivers of renal calciumphosphorus handling, such as FGF-23 and Klotho, were not measured in the present study. Primary hyperparathyroidism could not be excluded completely, because parathyroid images were not available. Universal consensus on the target fragments, analytic method, and reference value of measurement for these two biomarkers is still in debate. Further investigation should be warranted to elucidate the interplay of FGF-23, Klotho, vitamin D, and parathyroid axis with the risk of CKD.

In conclusion, this community-based study confirmed the association of serum levels of PTH and hyperparathyroidism with the risk of CKD. The serum levels of PTH increased in parallel with albuminuria but inversely with eGFR. Using a sample of the general Taiwanese population, the current study could provide a description of the prevalence of elevated serum intact PTH levels across a broad spectrum of kidney functions. This significant association with low eGFR was independent of dietary intake of calcium and serum levels of calcium, phosphorus, and $25(\mathrm{OH})$ D. Given its association with adverse outcomes in CKD, reducing serum intact $\mathrm{PTH}$ levels may be an important goal for improving CKD-MBD and may be potentially helpful for CKD prevention. The findings of the present study offer an insight for a further well-designed prospective study to clarify the causal relationship of PTH and $25(\mathrm{OH})$ vitamin D with renal function and risk of CKD in the general population.

\section{Competing Interests}

The authors declare no competing interests regarding the publication of this paper.

\section{Acknowledgments}

This study was sponsored by the Keelung Chang Gung Memorial Hospital Research Projects (CRRPG2B0134, CMRPG2C0342, and CMRPG2C0093).

\section{References}

[1] R. T. Gansevoort, R. Correa-Rotter, B. R. Hemmelgarn et al., "Chronic kidney disease and cardiovascular risk: epidemiology, mechanisms, and prevention," The Lancet, vol. 382, no. 9889, pp. 339-352, 2013.
[2] C. P. Wen, T. Y. D. Cheng, M. K. Tsai et al., "All-cause mortality attributable to chronic kidney disease: a prospective cohort study based on 462293 adults in Taiwan," The Lancet, vol. 371, no. 9631, pp. 2173-2182, 2008.

[3] USRDS, Annual Data Report: Epidemiology of Kidney Disease in the United States, National Institutes of Health, National Institute of Diabetes and Digestive and Kidney Diseases, Bethesda, Md, USA, 2016.

[4] G. Thomas, A. R. Sehgal, S. R. Kashyap, T. R. Srinivas, J. P. Kirwan, and S. D. Navaneethan, "Metabolic syndrome and kidney disease: a systematic review and meta-analysis," Clinical Journal of the American Society of Nephrology, vol. 6, no. 10, pp. 2364-2373, 2011.

[5] I. Al Mheid, R. Patel, J. Murrow et al., "Vitamin D status is associated with arterial stiffness and vascular dysfunction in healthy humans," Journal of the American College of Cardiology, vol. 58, no. 2, pp. 186-192, 2011.

[6] I. Mozos and O. Marginean, "Links between Vitamin D deficiency and cardiovascular diseases," BioMed Research International, vol. 2015, Article ID 109275, 12 pages, 2015.

[7] J. N. Artaza, R. Mehrotra, and K. C. Norris, "Vitamin D and the cardiovascular system," Clinical Journal of the American Society of Nephrology, vol. 4, no. 9, pp. 1515-1522, 2009.

[8] P. Prasad and A. Kochhar, "Interplay of vitamin D and metabolic syndrome: a review," Diabetes \& Metabolic Syndrome: Clinical Research \& Reviews, vol. 10, no. 2, pp. 105-112, 2016.

[9] D. Feldman, A. V. Krishnan, S. Swami, E. Giovannucci, and B. J. Feldman, "The role of vitamin D in reducing cancer risk and progression," Nature Reviews Cancer, vol. 14, no. 5, pp. 342-357, 2014.

[10] L. Zhang, S. Wang, X. Che, and X. Li, "Vitamin D and lung cancer risk: a comprehensive review and meta-analysis," Cellular Physiology and Biochemistry, vol. 36, no. 1, pp. 299-305, 2015.

[11] N. Agmon-Levin, E. Theodor, R. M. Segal, and Y. Shoenfeld, "Vitamin D in systemic and organ-specific autoimmune diseases," Clinical Reviews in Allergy and Immunology, vol. 45, no. 2, pp. 256-266, 2013.

[12] K. de Haan, A. B. J. Groeneveld, H. R. H. de Geus, M. Egal, and A. Struijs, "Vitamin D deficiency as a risk factor for infection, sepsis and mortality in the critically ill: systematic review and meta-analysis," Critical Care, vol. 18, no. 6, article 660, 2014.

[13] A. Nykjaer, D. Dragun, D. Walther et al., "An endocytic pathway essential for renal uptake and activation of the steroid $25-(\mathrm{OH})$ vitamin D3," Cell, vol. 96, no. 4, pp. 507-515, 1999.

[14] A. Nykjaer, J. C. Fyfe, R. Kozyraki et al., "Cubilin dysfunction causes abnormal metabolism of the steroid hormone $25(\mathrm{OH})$ vitamin $\mathrm{D}_{3}$," Proceedings of the National Academy of Sciences of the United States of America, vol. 98, no. 24, pp. 13895-13900, 2001.

[15] W. Al-Badr and K. J. Martin, "Vitamin D and kidney disease," Clinical Journal of the American Society of Nephrology, vol. 3, no. 5, pp. 1555-1560, 2008.

[16] J. Park, S. Ryu, M. Han, and S. Choi, "The Association of Vitamin D With Estimated Glomerular Filtration Rate and Albuminuria: 5th Korean National Health and Nutritional Examination Survey 2011-2012," Journal of Renal Nutrition, vol. 26, no. 6, pp. 360-366, 2016.

[17] B. Satirapoj, P. Limwannata, A. Chaiprasert, O. Supasyndh, and P. Choovichian, "Vitamin D insufficiency and deficiency with stages of chronic kidney disease in an Asian population," BMC Nephrology, vol. 14, article 206, 2013. 
[18] I. Guessous, W. McClellan, D. Kleinbaum et al., "Comparisons of serum vitamin d levels, status, and determinants in populations with and without chronic kidney disease not requiring renal dialysis: a 24-hour urine collection population-based study," Journal of Renal Nutrition, vol. 24, no. 5, pp. 303-312, 2014.

[19] I. Guessous, W. McClellan, D. Kleinbaum et al., "Serum 25hydroxyvitamin D level and kidney function decline in a Swiss general adult population," Clinical Journal of the American Society of Nephrology, vol. 10, no. 7, pp. 1162-1169, 2015.

[20] G. Zhao, E. S. Ford, C. Li, P. M. Kris-Etherton, T. D. Etherton, and L. S. Balluz, "Independent associations of serum concentrations of 25-hydroxyvitamin D and parathyroid hormone with blood pressure among US adults," Journal of Hypertension, vol. 28, no. 9, pp. 1821-1828, 2010.

[21] M. H. Grønhøj, O. Gerke, H. Mickley et al., "Associations between calcium-phosphate metabolism and coronary artery calcification; a cross sectional study of a middle-aged general population," Atherosclerosis, vol. 251, pp. 101-108, 2016.

[22] P. Muntner, T. M. Jones, A. D. Hyre et al., "Association of serum intact parathyroid hormone with lower estimated glomerular filtration rate," Clinical Journal of the American Society of Nephrology, vol. 4, no. 1, pp. 186-194, 2009.

[23] National Kidney Foundation, "K/DOQI clinical practice guidelines for chronic kidney disease: evaluation, classification and stratification," American Journal of Kidney Diseases, vol. 39, supplement 1, pp. S1-S266, 2002.

[24] I.-W. Wu, M.-J. Hung, Y.-C. Chen, W.-J. Cherng, and M.-S. $\mathrm{Wu}$, "High body mass index is not associated with coronary artery disease in angina patients with chronic kidney disease: a coronary angiography study," American Journal of the Medical Sciences, vol. 336, no. 4, pp. 303-308, 2008.

[25] L.-W. Chen, C.-Y. Chien, C.-W. Hsieh et al., "The associations between Helicobacter pylori infection, serum vitamin $\mathrm{D}$, and metabolic syndrome: a community-based study," Medicine, vol. 95, no. 18, Article ID e3616, 2016.

[26] I.-W. Wu, K.-H. Hsu, C.-C. Lee et al., "Re-evaluating the predictive roles of metabolic complications and clinical outcome according to eGFR levels-a four-years prospective cohort study in Taiwan," BMC Nephrology, vol. 14, no. 1, article 92, 2013.

[27] E. Theodoratou, I. Tzoulaki, L. Zgaga, and J. P. A. Ioannidis, "Vitamin D and multiple health outcomes: umbrella review of systematic reviews and meta-analyses of observational studies and randomised trials," BMJ, vol. 348, Article ID g2035, 2014.

[28] C. A. Keyzer, H. J. Lambers-Heerspink, M. M. Joosten et al., "Plasma vitamin D level and change in albuminuria and eGFR according to sodium intake," Clinical Journal of the American Society of Nephrology, vol. 10, no. 12, pp. 2119-2127, 2015.

[29] Y. J. Tak, J. G. Lee, S. H. Song et al., "The relationship between the level of serum 25-hydroxyvitamin D and renal function in patients without chronic kidney disease: a cross-sectional study," Journal of Renal Nutrition, vol. 25, no. 2, pp. 88-96, 2015.

[30] M. F. Holick, "Vitamin D status: measurement, interpretation, and clinical application," Annals of Epidemiology, vol. 19, no. 2, pp. 73-78, 2009.

[31] E. Fischer, A. Hannemann, R. Rettig et al., "A high aldosterone to renin ratio is associated with high serum parathyroid hormone concentrations in the general population," Journal of Clinical Endocrinology and Metabolism, vol. 99, no. 3, pp. 965971, 2014.
[32] P. Evenepoel, J. Bover, and P. Ureña Torres, "Parathyroid hormone metabolism and signaling in health and chronic kidney disease," Kidney International, vol. 90, no. 6, pp. 1184-1190, 2016.

[33] G. Rashid, J. Bernheim, J. Green, and S. Benchetrit, "Parathyroid hormone stimulates endothelial expression of atherosclerotic parameters through protein kinase pathways," American Journal of Physiology-Renal Physiology, vol. 292, no. 4, pp. F1215-F1218, 2007.

[34] J.-S. Shao, S.-L. Cheng, N. Charlton-Kachigian, A. P. Loewy, and D. A. Towler, "Teriparatide (human parathyroid hormone (1-34)) inhibits osteogenic vascular calcification in diabetic low density lipoprotein receptor-deficient mice," Journal of Biological Chemistry, vol. 278, no. 50, pp. 50195-50202, 2003.

[35] G. Kontogeorgos, P. Trimpou, C. M. Laine, G. Oleröd, A. Lindahl, and K. Landin-Wilhelmsen, "Normocalcaemic, vitamin D-sufficient hyperparathyroidism-high prevalence and low morbidity in the general population: a long-term follow-up study, the WHO MONICA project, Gothenburg, Sweden," Clinical Endocrinology, vol. 83, no. 2, pp. 277-284, 2015.

[36] V. M. Brandenburg, R. Kramann, H. Rothe et al., "Calcific uraemic arteriolopathy (calciphylaxis): data from a large nationwide registry," ndt: Nephrology Dialysis Transplantation, vol. 32, no. 1, pp. 126-132, 2017.

[37] C. M. Sturgeon, S. Sprague, A. Almond et al., "Perspective and priorities for improvement of parathyroid hormone (PTH) measurement-a view from the IFCC Working Group for PTH," Clinica Chimica Acta, 2016.

[38] J. Souberbielle, F. Brazier, M. Piketty, C. Cormier, S. Minisola, and E. Cavalier, "How the reference values for serum parathyroid hormone concentration are (or should be) established?" Journal of Endocrinological Investigation, pp. 1-16, 2016.

[39] T. Isakova, "Racial differences in parathyroid hormone levels in CKD," Nephrology Dialysis Transplantation, vol. 27, no. 7, pp. 2616-2617, 2012.

[40] M. W. Taal, V. Thurston, N. J. McIntyre, R. J. Fluck, and C. W. McIntyre, "The impact of vitamin D status on the relative increase in fibroblast growth factor 23 and parathyroid hormone in chronic kidney disease," Kidney International, vol. 86, no. 2, pp. 407-413, 2014.

[41] I. Guessous, O. Bonny, F. Paccaud et al., "Serum calcium levels are associated with novel cardiometabolic risk factors in the population-based CoLaus study," PLoS ONE, vol. 6, no. 4, Article ID e18865, 2011.

[42] L. Mateus-Hamdan, O. Beauchet, Y. Rolland, A.-M. Schott, and C. Annweiler, "Association of calcium concentration with pulse pressure in older women: Data from A Large Population-based Multicentric Study," Journal of Nutrition, Health \& Aging, vol. 18, no. 3, pp. 323-329, 2014. 


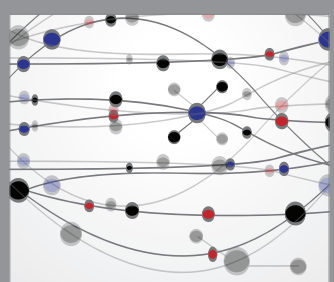

The Scientific World Journal
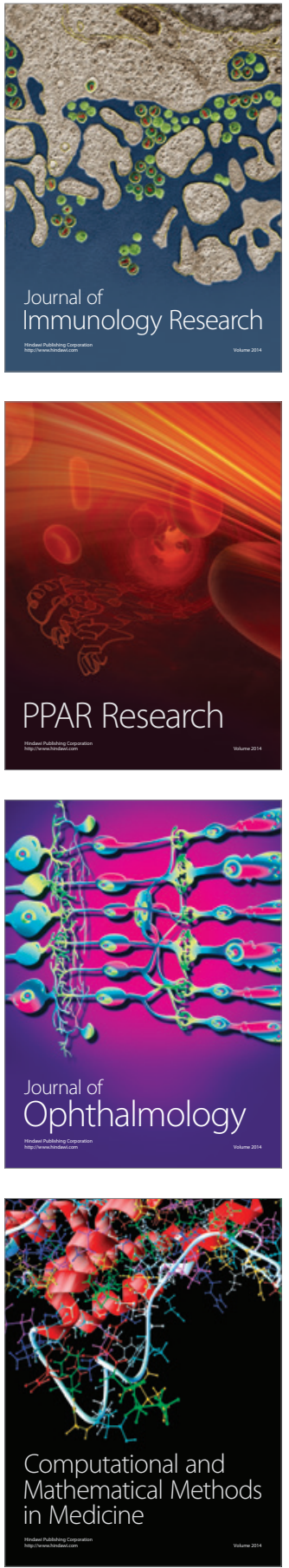

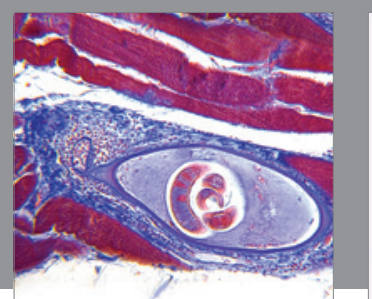

Gastroenterology Research and Practice
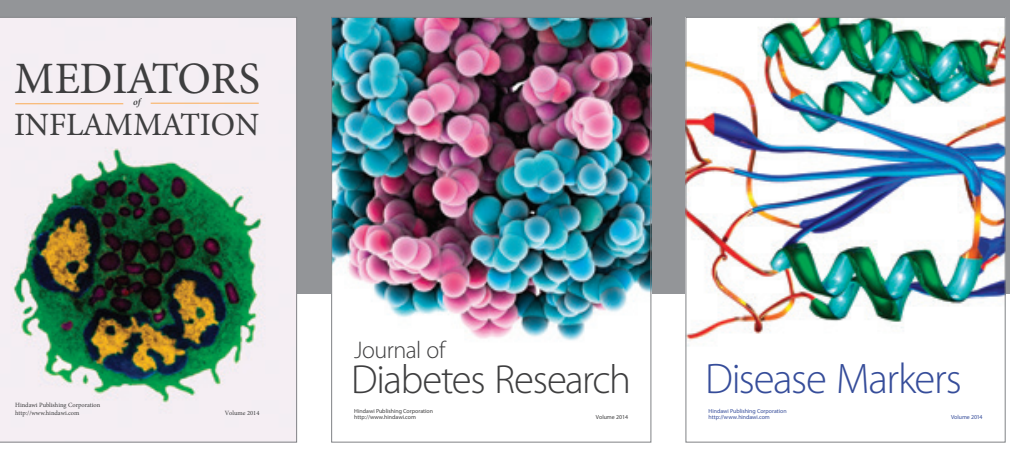

Disease Markers

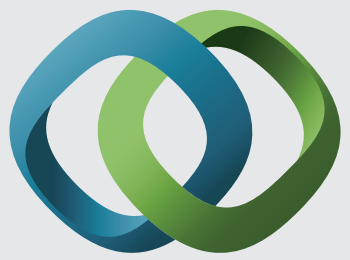

\section{Hindawi}

Submit your manuscripts at

https://www.hindawi.com
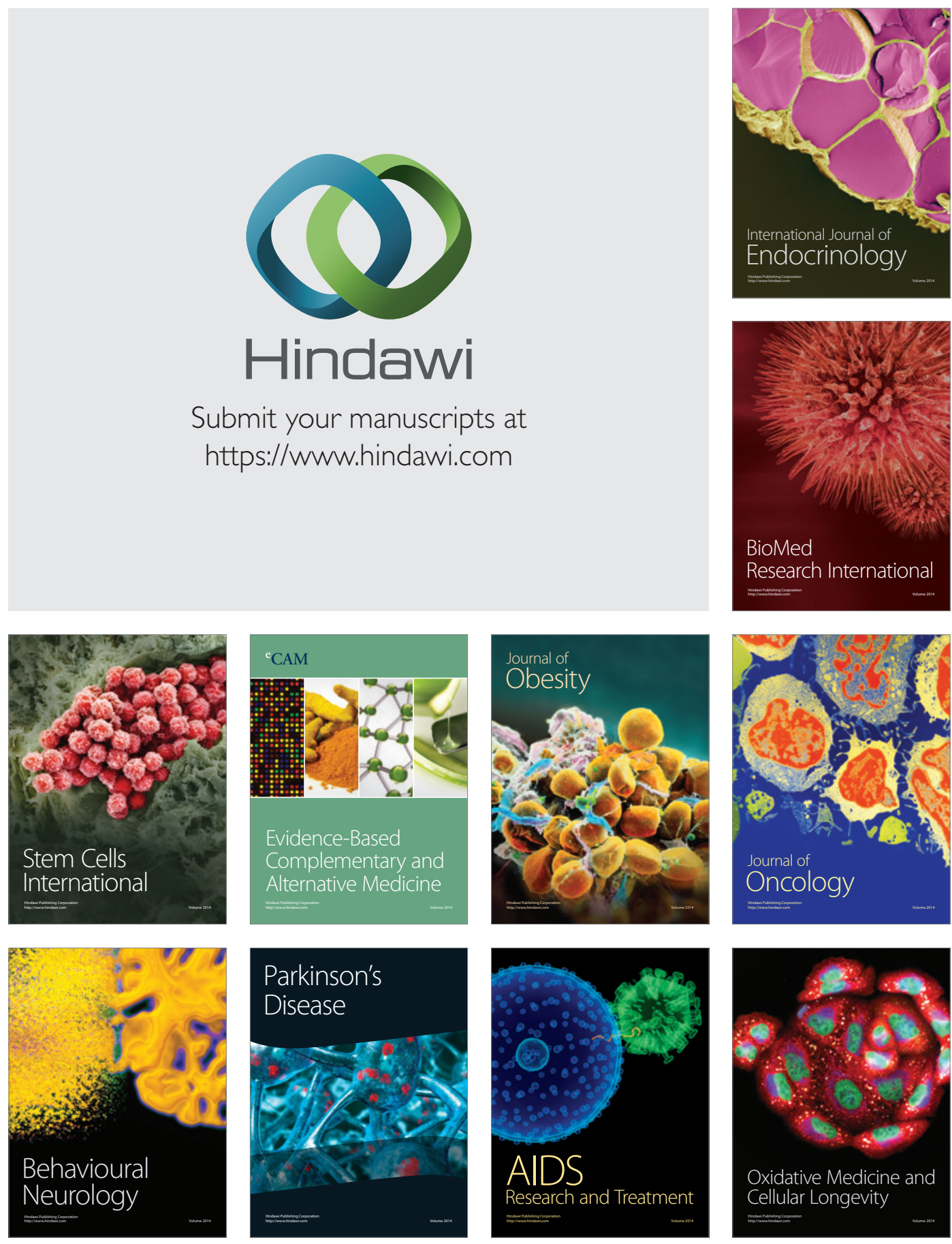